\title{
Biomatériaux vasculaires: du génie biologique et médical au génie tissulaire
}

Anne Meddahi-Pellé, Isabelle Bataille, Pascale Subra, Didier Letourneur

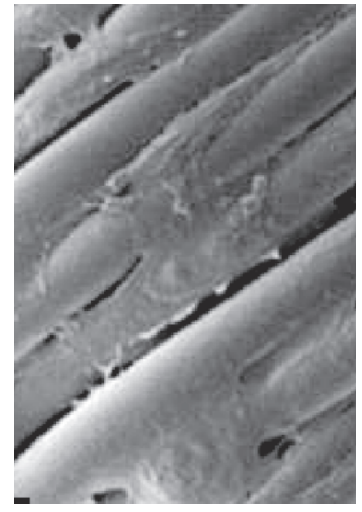

utile de rappeler quelques définitions pour mieux cerner ce vaste domaine et ses applications (Tableau 1), et présenter ensuite quelques axes récents sur les recherches en biomatériaux vasculaires.

\section{Biomatériaux et génie tissulaire : définitions}

La Conférence de Chester de la Société Européenne des Biomatériaux en 1986 a retenu pour les biomatériaux la définition suivante: «matériaux non vivants utilisés dans un dispositif médical destinés à interagir avec les systèmes biologiques». La notion de biocompatibilité (capacité d'un matériau à être utilisé avec une réponse de l'hôte appropriée dans une application spécifique) peut y être associée. Pour que le biomatériau corresponde à ces critères, il est généralement admis que la durée de contact avec les tissus vivants dépasse quelques heures, ce qui exclut les produits pharmaceutiques, mais intègre les systèmes de libération contrôlée de principes actifs. Le concept de génie tissulaire (tissue engineering) est apparu plus récemment (Figure I). II 
intègre l'ensemble des technologies utilisant des cellules vivantes ou des biomatériaux (synthétiques ou naturels) dans le but de reconstruire ou régénérer des tissus et organes humains, de remplacer un organe déficient ou de modifier des gènes de l'organisme. Le domaine du génie tissulaire est en pleine émergence face à une demande toujours croissante de substituts de tissus, du déficit d'organes pour les transplantations, des limitations des xénotransplantations et des incertitudes techniques et éthiques liées au clonage. Des produits issus du génie tissulaire ont ainsi déjà vu le jour, comme les peaux artificielles (traitement des brûlés) ou les tests pharmaco-dermatologiques. Certains aspects du génie tissulaire ont été développés dans un précédent numéro de médecine/sciences [1]. Nous illustrerons plus particulièrement notre propos à travers l'exemple du génie tissulaire appliqué à la problématique des prothèses vasculaires.

\section{Problématique médicale des prothèses vasculaires}

L'incidence des maladies cardiovasculaires d'origine athérosclérotique demeure un problème majeur en santé publique. Malgré le développement de techniques curatives endovasculaires (angioplastie et endoprothèse vasculaire ou stent) (Figure 2A), la chirurgie reste nécessaire chez un nombre élevé de patients. Le remplacement vasculaire se fait alors de manière autologue par une veine ou parfois une artère ou, lorsque les patients n'ont pas de capital vasculaire suffisant, par une prothèse. Les techniques de remplacement vasculaire par des prothèses synthétiques comme le PTFEe (polytétrafluoroéthylène expansé) ou le Dacron ${ }^{\circledR}$ (polyéthylène téréphtalate) (Figure 2B-D) sont utilisées avec succès pour des remplacements d'artères de gros calibre malgré les risques d'infection. Pour les remplacements artériels de petit calibre (< $6 \mathrm{~mm}$ de diamètre) ou pour les remplacements veineux, les résultats ne sont pas favorables car la diminution du flux vasculaire et la réponse hyperplasique intimale péri-anastomotique contribuent à leur occlusion. Le développement de prothèses présentant une face interne non thrombogène et facilement implantable, reste un but à atteindre. Le génie tissulaire apparaît alors comme une solution alternative permettant d'obtenir des substituts de tissus ou d'organes.

Différentes approches ont été développées au cours des dernières années. La première consiste à maintenir la perméabilité vasculaire pour permettre le développement à la surface interne de la prothèse d'un endothélium vasculaire assurant le caractère non thrombogène de la surface. Cependant, l'endothélialisation spontanée reste limitée chez l'homme, contrairement aux résultats obtenus chez l'animal, sans que l'on comprenne bien les raisons de cette différence.

La seconde approche consiste à obtenir par génie tissulaire, selon des techniques in vitro ou in vivo, un greffon vasculaire autologue présentant des caractéristiques structurales et fonctionnelles se rapprochant de celles d'un vaisseau natif [2, $3]$. Nous présentons ainsi certaines approches dans le choix des surfaces et des cellules à utiliser.

\section{Domaines}

Cardiovasculaire

Chirurgie plastique et reconstructive

Chirurgie orthopédique

Endocrinologie

Odontologie et stomatologie

Ophtalmologie

Radiologie et imagerie

Urologie et néphrologie

Autres ou applicables à plusieurs domaines

\section{Applications}

Valves cardiaques, matériel pour circulation extra-corporelle, cœur artificiel, assistance ventriculaire, stimulateurs cardiaques, prothèses et endoprothèses (stents) vasculaires, matériels pour angioplastie luminale, cathéters

Matériaux et implants pour chirurgie esthétique, drains de chirurgie, colles tissulaires, peau artificielle, sutures résorbables

Prothèses articulaires (hanche, coude, genou, poignet), orthèses, ligaments et tendons artificiels, cartilage, matériel de remplacement ou comblement osseux, chirurgie du rachis, réparation de fractures (vis, plaques, clous, broches)

Pancréas artificiel, pompes portables et implantables

Matériaux de restauration, comblement dentaire et osseux, traitements prophylactiques, orthodontie, traitements du parodonte et de la pulpe, implants, reconstruction maxillo-faciale

Implants, lentilles, coussinets de récupération, produits visqueux de chambre postérieure Produits de contraste, produits pour embolisation, produits pour radiologie interventionelle Dialyseurs, poches, cathéters et tubulures pour dialyse, rein artificiel portable, prothèses, matériaux pour traitement de l'incontinence

Systèmes de libération contrôlée de médicaments ou de gènes, biocapteurs, encapsulation cellulaire, néo-intestin

Tableau I. Domaines d'application des biomatériaux. 


\section{Modification des matériaux par des composés d'origine biologique favorisant l'adhérence cellulaire}

\section{Protéines de la matrice extracellulaire}

Le succès clinique d'une prothèse est fortement tributaire de la qualité de l'adhérence des cellules à la surface du matériau, processus de base influençant directement la croissance, la différenciation et la migration des cellules. L'étape-clé se situe à l'échelle des adsorptions moléculaires et une modification chimique de la surface prothétique peut permettre de se rapprocher des caractéristiques de la matrice extracellulaire. Un moyen de rendre une surface synthétique apte à favoriser l'adhérence cellulaire est de fixer sur cette surface des constituants de la matrice extracellulaire. La technique classique consistait en l'imprégnation de la prothèse en Dacron ${ }^{\circledR}$ par du sang total, du plasma ou du sérum qui permettait d'obtenir une couche protéique nécessaire au développement des cellules endothéliales. De nombreux composés matriciels ont été utilisés pour augmenter l'attachement et la prolifération des cellules endothéliales. L'imprégnation des prothèses avec des colles à base de fibrine libérant des facteurs de croissance comme le FGF-1 (fibroblast growth factor-1) ou le VEGF (vascular endothelial growth factor) favorise la prolifération des cellules endothéliales [4]. Des stents en Nitinol (alliage nickel/titane) recouverts de fibronectine sont plus rapidement ré-endothélialisés in

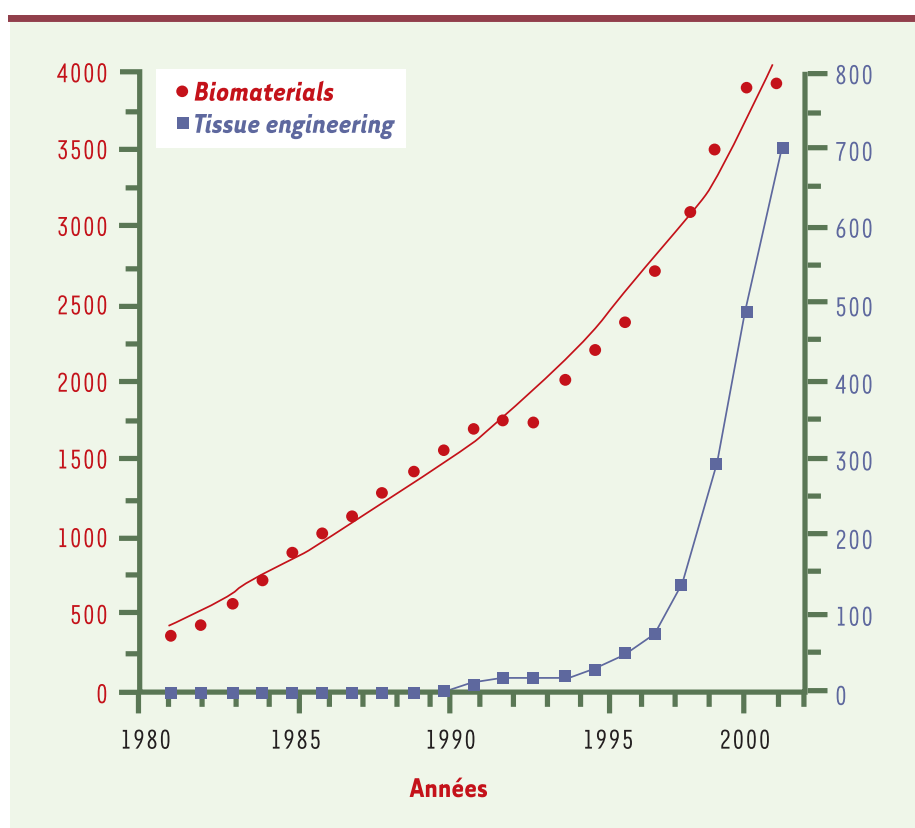

Figure 1. Citations annuelles depuis 1982 des termes Biomaterials (en rouge) et Tissue engineering (en bleu) dans la base de données Pubmed de la United States National Library of Medicine (http://www.ncbi.nlm.nih.gov/). Le nombre de citations est donné à titre d'exemple et ne prend en compte que les revues biomédicales, les articles concernant les caractères chimique ou physique des matériaux étant exclus. vitro. L'hirudine ou l'héparine, composés connus pour leurs propriétés antithrombotiques ont également été testés [5]. Les collagènes de type II et III recouvrant des films de Dacron ${ }^{\circledR}$ modifiés par greffage covalent d'acide polyacrylique augmentent l'attachement et la prolifération de cellules vasculaires [6]. Néanmoins, ces protéines favorisent l'adhérence plaquettaire, donc les phénomènes de thrombose, et augmentent le risque infectieux [7]. Enfin, l'utilisation actuelle de protéines d'origine humaine ou animale pour le recouvrement de prothèses pose le problème du risque de transmission d'agents infectieux. Certaines stratégies s'orientent donc vers des matériaux de synthèse ne contenant pas de composés biologiques.

\section{Peptides d'attachement}

La plupart des protéines de la matrice extracellulaire contiennent parmi leurs séquences oligopeptidiques actives une séquence tripeptidique Arg-Gly-Asp à laquelle sont attribuées des propriétés d'adhérence cellulaire. Ce tripeptide possède une forte affinité pour les récepteurs cellulaires de la superfamille des intégrines, qui font partie des CAM (cell adhesion molecules) situées à la surface des cellules. L'équipe de C. Baquey (Inserm U.443), en étudiant l'adhérence de cellules endothéliales sur une surface modèle de silice où ont été fixés de façon covalente des peptides contenant la séquence Arg-Gly-Asp [8], a mis en évidence une amélioration de l'adhérence cellulaire. Des effets similaires ont été constatés après modification de gels d'alginate, hétéropolysaccharide ne possédant pas de propriétés intrinsèques d'ancrage des cellules [9]. Une augmentation de l'adhérence des fibroblastes cardiaques est obtenue sur une surface de silicone modifiée par fixation covalente du peptide Gly-Arg-Gly-Asp-Ser-Pro [10]. Certains polymères biodégradables comme l'acide polylactique présentent un grand intérêt pour le recouvrement des endoprothèses métalliques (Figure 2A), mais sont dépourvus de fonctions chimiques permettant le greffage direct de peptides Arg-Gly-Asp. Si ce peptide est greffé sur un polymère anionique comme la polylysine, puis adsorbé à la surface cationique du polylactide, l'étalement de cellules endothéliales d'aorte bovine est augmenté [11]. L'influence de la structure du peptide a été examinée, les peptides cycliques paraissant les plus efficaces sur les activités promotrices d'adhérence, probablement du fait d'une meilleure conformation du site d'adhérence [12]. Cette stratégie est également utilisée pour des biomatériaux non vasculaires [13].

\section{Modifications physiques ou chimiques de la surface des matériaux}

La surface des matériaux peut être modifiée par des méthodes physiques ou chimiques. Nous avons modifié des particules sphériques de polystyrène par introduc- 
tion de groupements sulfate sur la chaîne macromoléculaire. Ces supports permettent de retenir les facteurs de croissance FGF-1 et FGF-2 et servent de matrice synthétique pour la culture des cellules endothéliales bovines grâce à leur capacité à fixer les protéines matricielles (Figure 3). Une autre approche largement utilisée consiste en un recouvrement (coating) de la surface du matériau par un polymère adapté. Ce recouvrement peut être réalisé sur des prothèses en Dacron ${ }^{\circledast}$ et sur des stents métalliques avec délivrance locale de cellules vasculaires ou de gènes [14]. En fonction des utilisations, le polymère de recouvrement peut être dégradable et inclure un agent pharmacologique. L'incorporation d'un principe actif dans le polymère de recouvrement est ainsi largement développée dans le domaine des stents [14]. Les premiers essais cliniques sont très prometteurs même si les résultats à long terme restent à évaluer [15]. D’autres développements consisteraient à introduire des agents plus spécifiques actifs sur la prolifération des cellules musculaires lisses $[16,17]$ et sur les phénomènes inflammatoires [18].

\section{Avec quelles cellules effectuer la colonisation cellulaire des matériaux vasculaires?}

Plusieurs types cellulaires sont envisageables pour la colonisation de biomatériaux vasculaires. L'approche classique de modification des matériaux prothétiques vasculaires consiste à réaliser une endothélialisation in vitro (Figure 2D). Deux stratégies sont utilisées: la première consiste à préparer une prothèse «endothélialisée» par isolement des cellules endothéliales, multiplication cellulaire, implantation in vitro sur la prothèse, endothélialisation de celle-ci et greffe; dans l'autre technique, la prothèse est ensemencée avec une densité cellulaire élevée et implantée en un seul temps [4]. La source cellulaire est le plus souvent veineuse, plus rarement artérielle. Dès 1984, M. Herring et al. présentaient les premiers résultats cliniques [19]. M. Deutsch et al. implantent des prothèses fémoropoplitées endothélialisées et observent un taux élevé de perméabilité à 3 et 7 ans ( $85 \%$ et $74 \%$ ) comparé aux prothèses non endothélialisées $(55 \%$ et $0 \%$ ) [20].
Cependant, les difficultés d'obtention des cellules endothéliales et la faible adhérence en flux limitent l'utilisation clinique de ces procédés. Les cellules une fois réimplantées doivent conserver leurs caractères phénotypiques pour présenter une surface antithrombogénique et réguler la vasomotricité et la prolifération des cellules musculaires lisses locales. L'utilisation de techniques de culture en flux permettent, par exemple, aux cellules endothéliales d'acquérir des fonctions nécessaires à la prise de la greffe [21]. Néanmoins le phénotype des cellules endothéliales peut être profondément modifié et le nombre de repiquages diminue l'adhérence, la prolifération cellulaire [22] et les caractéristiques fonctionnelles [23].

D’autres approches ont été utilisées, faisant appel à des cellules mésothéliales provenant des tissus graisseux, à la modification génique des cellules endothéliales ou à d'autres types cellulaires. L'ensemencement de cellules endothéliales génétiquement modifiées permet de disposer de cellules capables de proliférer localement, de présenter une meilleure résistance au flux et de produire localement des substances vasoactives (pour revue, voir [4]). Parmi les autres types cellulaires, les cellules

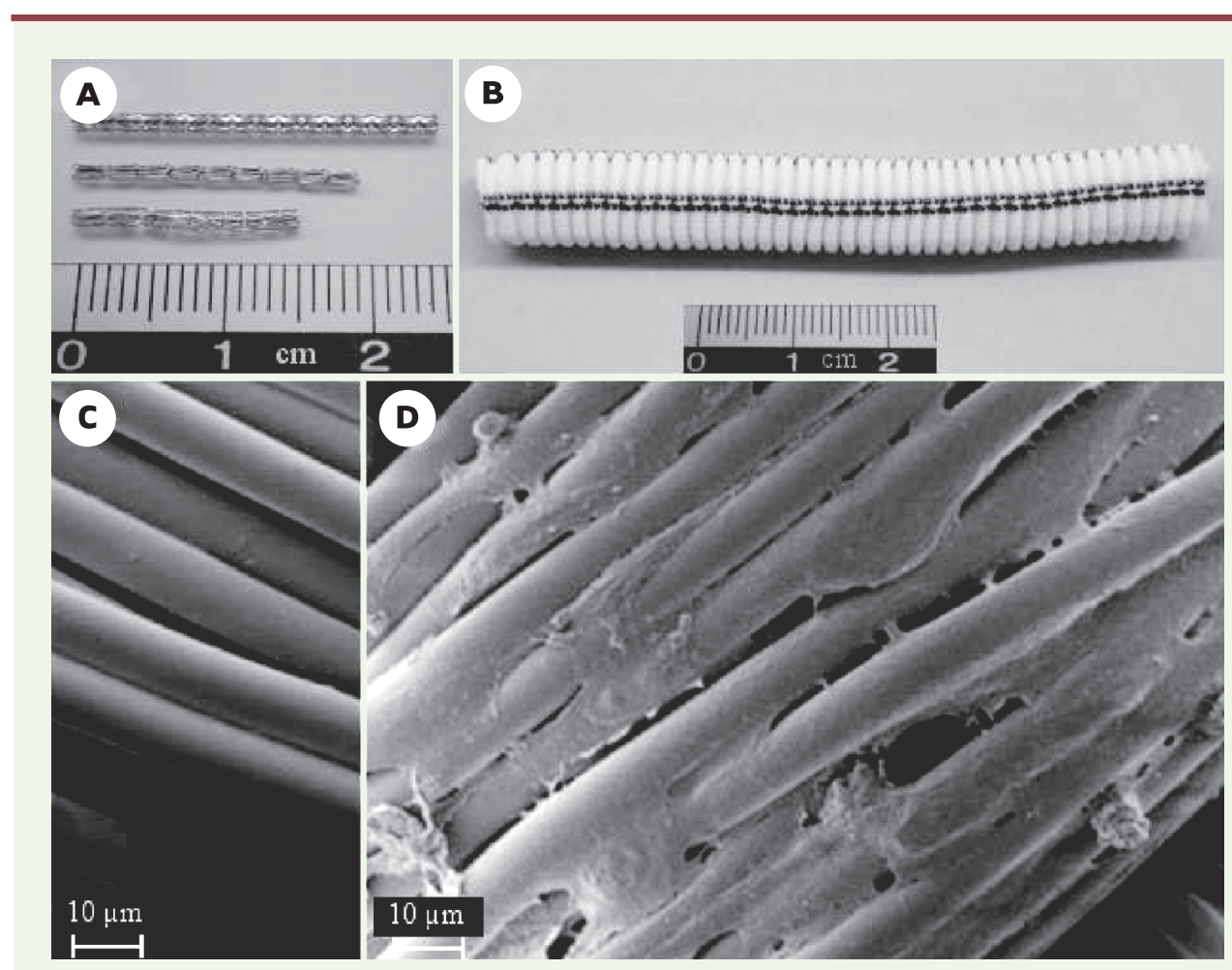

Figure 2. Exemple de prothèses vasculaires et de leur recouvrement. (A) Endoprothèses métalliques (stents). (B) Prothèse vasculaire en polyéthylène téréphtalate (PET ou Dacron ${ }^{\circledR}$ ). Microscopie électronique à balayage avant (C) et après $(D)$ recouvrement d'une prothèse en Dacron ${ }^{\circledast}$ par des polymères et croissance in vitro de cellules endothéliales humaines. 
souches progénitrices obtenues à partir de biopsies de moelle osseuse pourraient présenter une alternative intéressante, facilitant l'obtention d'un endothélium autologue [24]. S. Kaushal et al. ont ensemencé des cellules souches progénitrices circulantes sur des matrices décellularisées d'artères iliaques de porc et les ont implantées en remplacement d'artères carotides. Ces auteurs ont observé une perméabilité à 130 jours après implantation et des caractères de contractilité identiques à celles d'un vaisseau natif [21]. Des cellules souches cultivées sur un polymère biodégradable comme l'acide polyglycolique permettent d'obtenir une structure organisationnelle de type myofibroblastique identique à celle observée avec des cellules vasculaires [25].

Une approche différente utilisée par l'équipe de J.B. Michel (Inserm U.460) consiste en l'utilisation de cellules musculaires lisses vasculaires [26]. De production plus aisée, ces cellules, qui sont celles de la cicatrisation vasculaire, résistent mieux à l'arrachement au flux sanguin. L'implantation in vivo d'un tronçon aortique décellularisé ensemencé par des cellules musculaires lisses n'entraîne pas de problèmes thrombotiques [26]. Néanmoins, les travaux expérimentaux ont été réalisés sur des modèles animaux et le passage à une application clinique n'a pas encore été réalisé.

\section{Reconstitution de vaisseaux par génie tissulaire}

Deux voies de recherche se sont développées. La première consiste à fabriquer un vaisseau de substitution à partir de matériel purement biologique (culture cellulaire, tissus). La deuxième approche relève du concept de matériau hybride et associe dans une même unité fonctionnelle une composante cellulaire et une composante artificielle, si possible dégradable, permettant d'assurer une bonne tenue mécanique de l'ensemble. Dès 1953, ع.C. Pierce avait décrit l'implantation d'un tube fibrocollagénique vasculaire [27]. En raison de problèmes anévrysmaux rencontrés avec ce type de structure, il est nécessaire d'ajouter un squelette polymère pour renforcer la solidité de la structure finale. N. L'Heureux et al. [28] ont conçu un vaisseau in vitro en cultivant de manière séparée les diverses couches vasculaires: une couche de cellules musculaires lisses dans un gel de collagène est déposée autour d'un tuteur et une couche externe de cellules fibroblastiques est alors ensemencée. La couche de cellules endothéliales est appliquée ou non à l'intérieur [1]. R.T. Tranquillo et al. [29] montrent que des fibroblastes ensemencés dans un gel de collagène le long d'un tuteur permettent

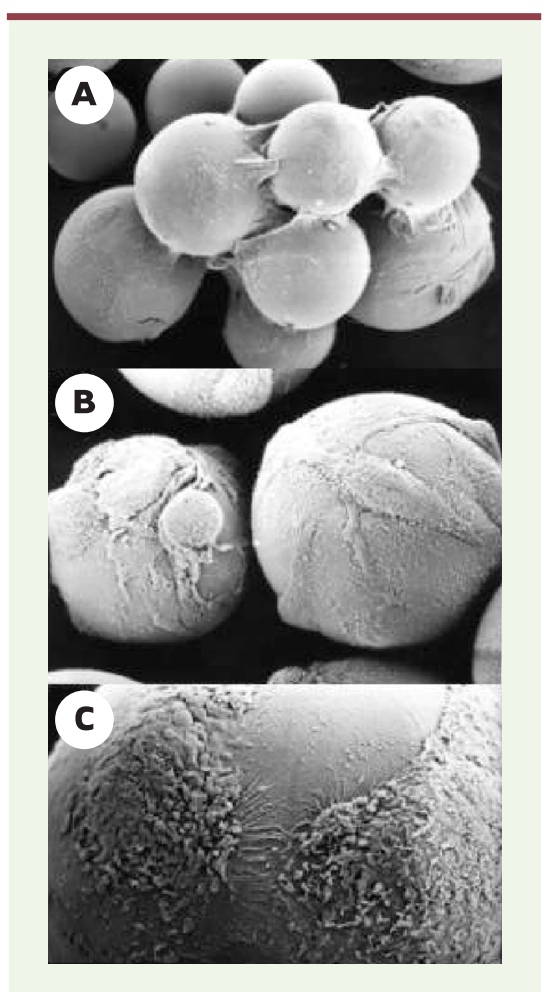

Figure 3. Microscopie électronique à balayage de billes de polystyrène greffé par des groupements sulfate. Les supports fonctionnalisés chimiquement permettent de favoriser l'adhérence et la croissance in vitro de cellules endothéliales. A. Grossissement X 500; B. Grossissement X $900 ;$ C. Grossissement X 3000 . la contraction du gel de collagène autour de ce mandrin avec une amélioration notable de la solidité de la construction et le maintien du diamètre interne. J.H. Campbell et al. insèrent des tubes de silastic dans la cavité péritonéale de rat [3] qui se recouvre de plusieurs couches de cellules myofibroblastiques, de collagène matriciel et d'une couche de cellules mésothéliales. Retournée en doigt de gant pour présenter la face mésothéliale au flux, cette formation reste perméable durant quatre mois et présente une structure histologique s'approchant de celle des vaisseaux et répondant aux substances vasoactives. $\varepsilon$. Allaire et al. [30] réticulent chimiquement une matrice aortique décellularisée et l'ensemencent avec des cellules musculaires lisses vasculaires. La matrice de collagène fibrillaire peut être obtenue à partir d'uretère bovin réticulé chimiquement [31], de fascia musculaire dorsal chez le lapin [32] ou de sous-muqueuse intestinale implantée en position artérielle [33]. Ces sources tissulaires semblent donc présenter un intérêt comme substitut vasculaire. Néanmoins, malgré l'obtention d'une structure histologique finale satisfaisante et quelque soit la technique utilisée, il reste le problème de la tenue mécanique du néovaisseau en flux et la possibilité de survenue d'anévrysmes pariétaux.

Les premiers vaisseaux hybrides obtenus par ingénierie tissulaire furent décris en 1986 par C.B. Weinberg et $\varepsilon$. Bell [34]. Des cellules musculaires lisses et des fibroblastes étaient cultivés dans des tubes formés par des treillis de Dacron ${ }^{\circledR}$ visant à donner une stabilité mécanique au système. Le vaisseau était ensuite ensemencé par des cellules endothéliales. Cependant, le matériau obtenu n'était pas capable de résister aux fortes pressions vasculaires in vivo. Pour améliorer la tenue mécanique, L.E. Niklason et al. [2, 35] ont créé des néovaisseaux en conditions dynamiques de flux. Des cellules musculaires lisses d'aorte bovine sont ensemencées dans un tube polymère biodégradable et cultivées en flux, puis, des cellules endothéliales bovines sont implantées. Le vaisseau obtenu présente une densité cellulaire et un contenu en collagène proches de ceux retrouvés in vivo, répond aux substances vasoactives, et résiste à des forces de pression de l'ordre de $2000 \mathrm{mmHg}$ et reste perméable quatre semaines après implantation chez le porc.

Le problème de cette approche réside dans la maîtrise des interactions entre les matériaux utilisés et le tissu vivant. Dans ce contexte, la poursuite des études sur les réactions du vivant à l'échelle moléculaire, cellulaire et tissulaire au contact d'un matériau 
hybride permettront des avancées dans la réalisation de nouveaux biomatériaux pour le génie tissulaire.

\section{Conclusions}

De nouvelles stratégies de recherche dans le domaine des biomatériaux font actuellement l'objet d'importantes études pluridisciplinaires impliquant des chirurgiens, des cardiologues, des radiologues, des chimistes, des biologistes, des pharmaciens, des mécaniciens et des physiciens. La connaissance des interactions entre les matériaux et les milieux vivants visent à une intégration dans (ou un remplacement par) les tissus naturels. Avec l'aide de biomatériaux appropriés et développés en partenariat industriel, on saura un jour implanter un cœur artificiel, mais aussi effectuer les réparations du tissu nerveux. Quel que soit le domaine, chirurgie, génie tissulaire, génie génétique, biotechnologie ou imagerie, l'ère des biomatériaux se poursuit ! $\nabla$

\section{REMERCIEMENTS}

Les travaux en génie tissulaire provenant de nos laboratoires ont été soutenus par l'Inserm, la Fondation Bettencourt-Schueller, la Fondation de l'Avenir, la Fondation de la Recherche Médicale, l'Université Paris 13 et le Ministère de la Recherche.

\section{SUMMARY}

Vascular biomaterials: From biomedical engineering to tissue engineering

Biomaterials are already widely used in medical sciences. The field of biomaterials began to shift to produce materials able to stimulate specific cellular responses at the molecular level. The combined efforts of cell biologists, engineers, materials scientists, mathematicians, geneticists, and clinicians are now used in tissue engineering to restore, maintain, or improve tissue functions or organs. This rapidly expanding approach combines the fields of material sciences and cell biology for the molecular design of polymeric scaffolds with appropriate 3D configuration and biological responses. Future developments for new blood vessels will require improvements in technology of materials and biotechnology together with the increased knowledge of the interactions between materials, blood, and living tissues. Biomaterials represent a crucial mainstay for all these studies. $\diamond$

\section{RÉFÉRENCES}

1. Auger FA. Le génie tissulaire: du rêve à la réalité. Med Sci (Paris) 2000; 16: 1324-31.

2. Niklason $L \varepsilon$, Gao J, Abbott WM, et al. Functional arteries grown in vitro. Science $1999 ; 284:$ 489-93.

3. Campbell JH, દfendy JL, Campbell GR. Novel vascular graft grown within recipient's own peritoneal cavity. Circ Res 1999; 85 : 1173-8.

4. Parikh SA, Edelman ER. Endothelial cell delivery for cardiovascular therapy. Adv Drug Deliv Rev 2000; 42: 139-61.

5. Kong X, Grabitz RG, van OW, et al. Effect of biologically active coating on biocompatibility of Nitinol devices designed for the closure of intra-atrial communications. Biomaterials 2002; 23: 1775-83.

6. Bisson I, Kosinski M, Ruault S, et al. Acrylic acid grafting and collagen immobilization on poly (ethylene terephthalate) surfaces for adherence and growth of human bladder smooth muscle cells. Biomaterials 2002; $23: 3149-58$.

7. Montdargent B, Letourneur D. Toward new biomaterials. Infect Control Hosp Epidemiol 2000; 21: 404-10.

8. Porte-Durrieu MC, Labrugere C, Villars F, et al. Development of RGD peptides grafted onto silica surfaces: XPS characterization and human endothelial cell interactions. J Biomed Mater Res 1999; 46: 368-75.

9. Rowley JA, Mooney DJ. Alginate type and RGD density control myoblast phenotype. J Biomed Mater Res 2002; 60: 217-23.

10. Lateef SS, Boateng S, Hartman TJ, et al. GRGDSP peptide-bound silicone membranes withstand mechanical flexing in vitro and display enhanced fibroblast adhesion. Biomaterials 2002; 23 : 3159-68.

11. Quirk RA, Chan WC, Davies MC, et al. Poly(L-lysine)-GRGDS as a biomimetic surface modifier for poly(lactic acid). Biomaterials 2001; 22: 865-72.

12. Verrier $S$, Pallu $S$, Bareille R, et al. Function of linear and cyclic RGD-containing peptides in osteoprogenitor cells adhesion process. Biomaterials 2002; 23: 585-96.

13. Jeschke B, Meyer J, Jonczyk A, et al. RGD-peptides for tissue engineering of articular cartilage. Biomaterials 2002; 23: 3455-63.

14. Nakayama Y, Ji-Youn K, Nishi S, et al. Development of high-performance stent: Gelatinous photogel-coated stent that permits drug delivery and gene transfer. J Biomed Mater Res 2001; 57: 559-66.

15. Sousa JE, Costa MA, Abizaid AC, et al. Sustained suppression of neointimal proliferation by sirolimus-eluting stents: one-year angiographic and intravascular ultrasound follow-up. Circulation 2001; 104: 2007-11.

16. Deux JF, Meddahi-Pelle A, Le Blanche AF, et al. Low molecular weight fucoidan prevents neointimal hyperplasia in rabbit iliac artery in-stent restenosis model. Arterioscler Thromb Vasc Biol 2002; 22: 1604-9.

17. Deux JF, Prigent-Richard S, d'Angelo G, et al. A chemically modified dextran inhibits smooth muscle cell growth in vitro and intimal in stent hyperplasia in vivo. J Vasc Surg 2002; 35: 973-81

18. Feldman LJ, Aguirre L, Ziol M, et al. Interleukin-10 inhibits intimal hyperplasia after angioplasty or stent implantation in hypercholesterolemic rabbits. Circulation $2000 ; 101: 908-16$.

19. Herring M, Gardner A, Glover J. Seeding human arterial prostheses with mechanically derived endothelium. The detrimental effect of smoking. J Vasc Surg 1984; 1: 279-89.

20. Deutsch M, Meinhart J, Fischlein T, et al. Clinical autologous in vitro endothelialization of infrainguinal ePTFE grafts in 100 patients: a 9-year experience. Surgery 1999; 126: 847-55.

21. Kaushal S, Amiel GE, Guleserian KJ, et al. Functionnal small-diameter neovessels created using endothelial progenitor cells expended ex vivo. Nat Med 2001; 7 : $1035-40$.

22. Radomski M, Jarell B, Pratt $K$, et al. Effects of in vitro aging on human endothelial cell adherence to dacron vascular graft materials. J Vasc Surg 1989; 47: 173-7.

23. Fournet-Bourguignon MP, Castedo-Delrieu M, Bidouard JP, et al. Phenotypic and functional changes in regenerated porcine coronary endothelial cells: Increased uptake of modified LDL and reduced production of NO. Circ Res 2000; 2000: 854-61.

24. Pittenger MF, Mackay AM, Beck SC. Multilineage potential of adult human mesenchymal stem cells. Science 1999; 284: 143-7.

25. Kadner A, Hoerstrup SP, Zund G, et al. A new source for cardiovascular tissue engineering: human bone marrow stromal cells. Eur J Cardiothorac Surg 2002; 21: $1055-60$.

26. Gomes D, Louedec L, Plissonnier D, et al. Endoluminal smooth muscle cell seeding limits intimal hyperplasia. J Vasc Surg 2001; 34: 707-15.

27. Pierce $\varepsilon C$. Autologous tissue tubes for aortic grafts in dogs. Surgery 1953; $33: 648$

28. L'Heureux $N$, Paquet $S$, Labbe $R$, et al. A completely biological tissue-engineered human blood vessel. FASEB J 1998; 12: 47-56.

29. Tranquillo RT, Girton TS, Bromberek BA, et al. Magnetically-oriented tissue equivalent tubes. Biomaterials 1995; $17: 349-53$.

30. Allaire $\varepsilon$, Guettier C, Bruneval P, et al. Cell-free arterial grafts: morphologic characteristics of aortic isografts, allografts, and xenografts in rats. J Vasc Surg 1994; 19: 446-56.

31. Field PL. The chemically treated bovine ureter. Clinical performance of a nouvel biological vascular prosthesis. Cardiovasc Surg 2003; 11:30-4.

32. Tsukagoshi T, Yenidunya MO, Sasaki $\varepsilon$, et al. Experimental vascular graft using small-caliber fascia-wrapped fibrocollagenous tube: short-term evaluation. J Reconstr Microsurg 1999; 15: 127-31.

33. Huynh T, Abraham G, Murray J, et al. Remodeling of an acellular collagen graft into a physiologically responsive neovessel. Nat Biotechnol 1999; 17: 1083-6.

34. Weinberg CB, Bell $\varepsilon$. A blood vessel model constructed from collagen and cultured vascular cells. Science 1986; 231: 397-400.

35. Niklason LE. Techview: medical technology. Replacement arteries made to order Science 1999 ; 286 : 1493-4.

TIRÉS À PART

A.Meddahi-Pellé 